\title{
MuCap: Muon Capture on the Proton
}

\author{
Malte Hildebrandt and Claude Petitjean ${ }^{\star}$
}

Paul Scherrer Institut, 5232 Villigen PSI, Switzerland

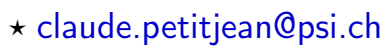

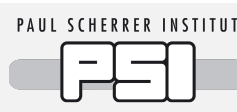

Review of Particle Physics at PSI

doi:10.21468/SciPostPhysProc.5

\begin{abstract}
The singlet muon capture rate $\Lambda_{S}$ on the proton $\mu^{-} p \rightarrow v_{\mu} n$ is determined in a high precision lifetime measurement. The main apparatus consists of a new hydrogen time projection chamber as muon detector, developed by PSI, surrounded by cylindrical wire chambers and a plastic scintillator hodoscope as electron detectors. The parameter $\Lambda_{S}$ is evaluated as the difference between the inverse $\mu p$ lifetime and that of the free $\mu^{+}$. The result $\Lambda_{S}^{\text {MuCap }}=\left(715.6 \pm 5.4^{\text {stat }} \pm 5.1^{\text {sys }}\right) \mathrm{s}^{-1}$ is in excellent agreement with the prediction of chiral perturbation theory $\Lambda_{S}^{\chi \mathrm{PT}}=(715.4 \pm 6.9) \mathrm{s}^{-1}$. From $\Lambda_{S}^{\mathrm{MuCap}}$ a recent analysis derives for the induced pseudoscalar coupling $g_{p}^{\text {MuCap }}=8.23 \pm 0.83$ whereas $\bar{g}_{p}^{\chi \mathrm{PT}}=8.25 \pm 0.25$.
\end{abstract}

Copyright M. Hildebrandt and C. Petitjean. This work is licensed under the Creative Commons Attribution 4.0 International License.

Published by the SciPost Foundation.
Received 13-01-2021

Accepted 31-03-2021

Published 06-09-2021

doi:10.21468/SciPostPhysProc.5.017

\subsection{Introduction}

Muon capture on the proton

$$
\mu^{-} p \rightarrow v_{\mu} n
$$

is a very important elementary process in weak interactions [1]. A measurement of the singlet capture rate $\Lambda_{S}$ is directly related to fundamental electroweak coupling constants $g_{A}$ and $g_{P}$. While $g_{A}$ is accurately known from measurements of the neutron lifetime, the induced pseudoscalar coupling $g_{P}$, can only be precisely determined from the muon capture rate. In low-energy chiral perturbation theory $(\chi \mathrm{PT}), g_{P}$ can be expressed as ${ }^{1}$

$$
g_{P}^{\chi \mathrm{PT}}\left(q^{2}\right)=\frac{2 m_{\mu} g_{\pi N N} f_{\pi}}{m_{\pi}^{2}-q^{2}}-\frac{1}{3} g_{A}(0) m_{\mu} m_{N} r_{A}^{2} .
$$

This leads to a theoretical prediction $[3,4]$ of

$$
\bar{g}_{P}^{\chi \mathrm{PT}} \equiv g_{P}^{\chi \mathrm{PT}}\left(q_{0}^{2}\right)=8.26 \pm 0.23,
$$

\footnotetext{
${ }^{1}$ The function $g_{P}\left(q^{2}\right) \equiv m_{\mu} / m_{N} F_{P}^{c c}\left(q^{2}\right)$ and $\bar{g}_{P}$ are defined in Section 5 [2].
} 
where $q_{0}^{2}=-0.88 m_{\mu}^{2}$. A precise measurement of $\Lambda_{S}$ represents therefore an important test of low-energy $\chi$ PT.

Historically, many experimental attempts to determine $\Lambda_{S}$ were already made in the 1960's at the leading accelerator labs to determine the $\mu p$ capture rate. These experiments resulted however only in a precision of $\sim 15 \%$, suffering mainly from two major challenges:

1) The output channel $v_{\mu} n$ consists only of neutral particles, where the $v_{\mu}$ escapes detection and the neutron is very difficult to be determined with high absolute precision. Modern experiments avoid this problem by using the lifetime method: instead of measuring absolute neutron rates, the disappearance rate of the muon, $\lambda_{\mu}$, is measured, i.e.

$$
\frac{d N_{\mu}}{d t}=N_{\mu} e^{-\lambda_{\mu} t}, \quad \lambda_{\mu}=\lambda_{0}+\Lambda_{S} .
$$

Here, $\lambda_{0}=0.455 \times 10^{6} \mathrm{~s}^{-1}$ is the decay constant ${ }^{2}$ of the free muon and $\Lambda_{S} \simeq 700 \mathrm{~s}^{-1}$ is just a small $\left(1.5 \times 10^{-3}\right)$ additional component of $\lambda_{\mu}$. Lifetime measurements therefore require high precision, i.e. large statistics. A first successful lifetime experiment was performed 1981 in Saclay [5] in a target with liquid hydrogen.

2) Negative muons in hydrogen quickly combine to neutral $(\mu p)$ atoms which behave like neutrons; they diffuse around and scatter with the surrounding nuclei. In collisions they can get easily transferred to heavier nuclei $(d, N, O)$ contained in the hydrogen. Moreover they can form the mesic molecule ( $p \mu p)$

$$
(\mu p) p \rightarrow(p \mu p)
$$

with a rate of about $\lambda_{p p \mu} \simeq 2 \times 10^{6} \mathrm{~s}^{-1}$. Two species of ( $\left.p \mu p\right)$ molecules exist, ortho-( $\left.p \mu p\right)$ and para- $(p \mu p)$. In the formation process, predominantly ortho-molecules are created, which eventually convert to the energetically lower para-molecule with rate $\lambda_{\mathrm{op}}$. Unfortunately, $\lambda_{\mathrm{op}}$ is not well known (theoretical value $\lambda_{\text {op }}^{\text {Th }}=(7.1 \pm 1.2) \times 10^{4} \mathrm{~s}^{-1}$ [6]). The capture rates differ strongly for the two states (for ortho- $(p \mu p) \sim 545 \mathrm{~s}^{-1}$, for para- $(p \mu p) \sim 215 \mathrm{~s}^{-1}$ ). This makes the interpretation of capture measurements in $(p \mu p)$ molecules difficult. This problem can be strongly reduced in hydrogen at low density, where the ( $p \mu p)$ formation rate is small.

In addition to these two major issues, isotope and chemical purities play an important role in the experiment. Natural hydrogen contains $\sim 150 \mathrm{ppm}$ deuterium nuclei. Muons in such a medium get quickly transferred to the heavier isotope

$$
(\mu p) d \rightarrow(\mu d) p .
$$

The $(\mu d)$ atoms are created at initial kinetic energy of $\sim 45 \mathrm{eV}$, and have a very large diffusion rate due to a $(\mu d)-p$ scattering minimum around $10 \mathrm{eV}$ (Ramsauer-Townsend effect). In collisions with deuterium nuclei they can form $(p \mu d)$ molecules leading to the muon catalyzed fusion

$$
(\mu p) d \rightarrow(p \mu d),(p \mu d) \rightarrow \mathrm{He}^{3}+\mu+5.5 \mathrm{MeV} .
$$

These processes would strongly interfere in a $\mu p$ capture measurement. Therefore, hydrogen depleted from deuterium (so called protium) has to be used. Furthermore, the protium must be kept at highest purity to avoid transfers to higher- $Z$ nuclei.

\footnotetext{
${ }^{2}$ denoted by $\Gamma_{\mu}$ in Section 5 [2]
} 


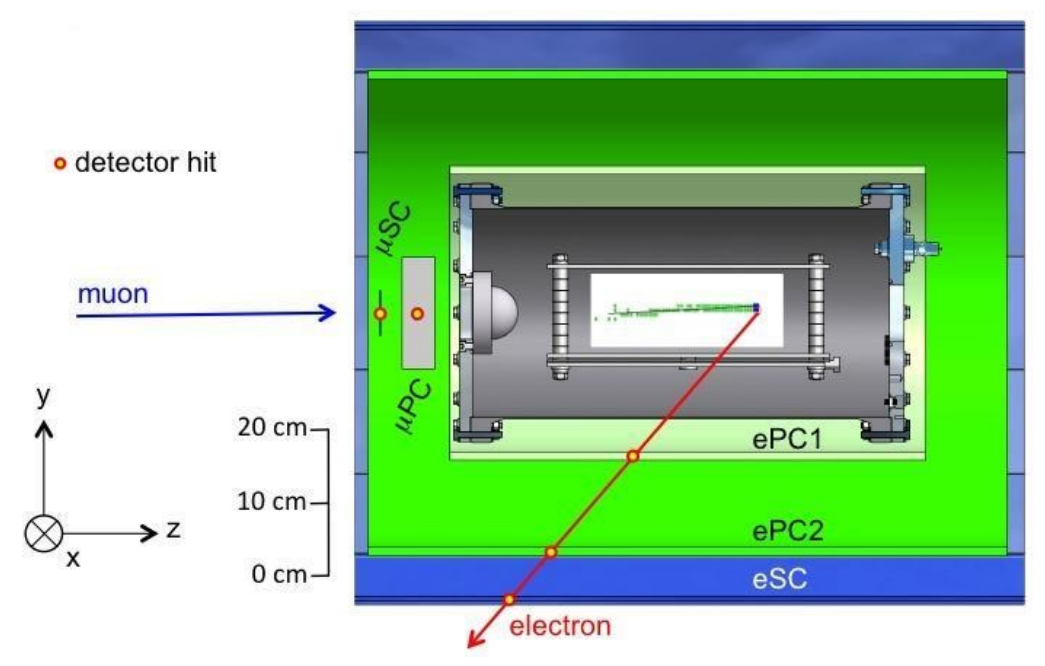

Figure 0.1: Cross section of the full MuCap apparatus with illustration of a typical event. Every muon was tracked individually to its stopping point. The electrons were tracked back to the muon stop location. Thanks to fiducial cuts, background from accidental electrons was suppressed to the $10^{-4}$ level.

\subsection{The MuCap experiment}

The MuCap experiment was proposed in 1997 with the goal to measure the singlet $\mu p$ capture rate $\Lambda_{S}$ to $1 \%$ precision which would then determine $g_{P}\left(q_{0}^{2}\right)$ to $\sim 6 \%$. This goal can be reached by a high precision measurement of the muon lifetime to the level $10^{-5}$ which requires a statistics of $\sim 10^{10}$ muon decay events.

Figure 0.1 shows a cross section of the MuCap experiment. The muon detector in the center consists of three components, a thin scintillator $\mu \mathrm{SC}$ providing the fast timing signal of the incoming muon, a wire chamber $\mu \mathrm{PC}$ and a time projection chamber TPC $[7,8]$ tracking the muon to the stopping point. The TPC is mounted inside an aluminium pressure vessel filled with 10 bar of ultra-pure protium gas. It acts simultaneously as muon stopping target and detector. The density of the protium gas is $\sim 1 \%$ of liquid hydrogen, thus avoiding the problems involved with meso-molecular processes. A special isotope separation column was constructed for MuCap [9] which removed deuterium to a negligible level. A special gas circulation system [10] was constructed using thermo-dynamical cycles and cryo-absorption by Zeolite filters for continuous cleaning of the protium gas. The system reduced impurity levels to values below $20 \mathrm{ppb}$.

The TPC was operated with a $2 \mathrm{kV} / \mathrm{cm}$ vertical electrical field. The electrons from the ionizing muon tracks - after drifting downwards to a multi-wire proportional chamber at the bottom - were collected in $x$ and $z$ coordinates. Combined with the drift time information ( $y$ coordinate) every muon track was reconstructed in three dimensions. After suitable fiducial cuts false muon stops were suppressed below the $10^{-5}$ level, necessary to keep the slope of the muon decay curve free from distortions. The electron detector consists of two cylindrical wire chambers ePC1, ePC2, and a plastic scintillation hodoscope eSC. The wire chambers - originally developed by PSI for the SINDRUM rare decay experiments, Section 7 [11] provide directional information for each electron track, while the scintillators yield the fast timing signal of the muon decay.

The anticipated precision was reached by collection of more than $10^{10}$ single good muon decay events. A significant boost of the statistics was achieved with help of the muon kicker [12] from the MuLan experiment [13] ('muons on request' method). The system transmitted single 


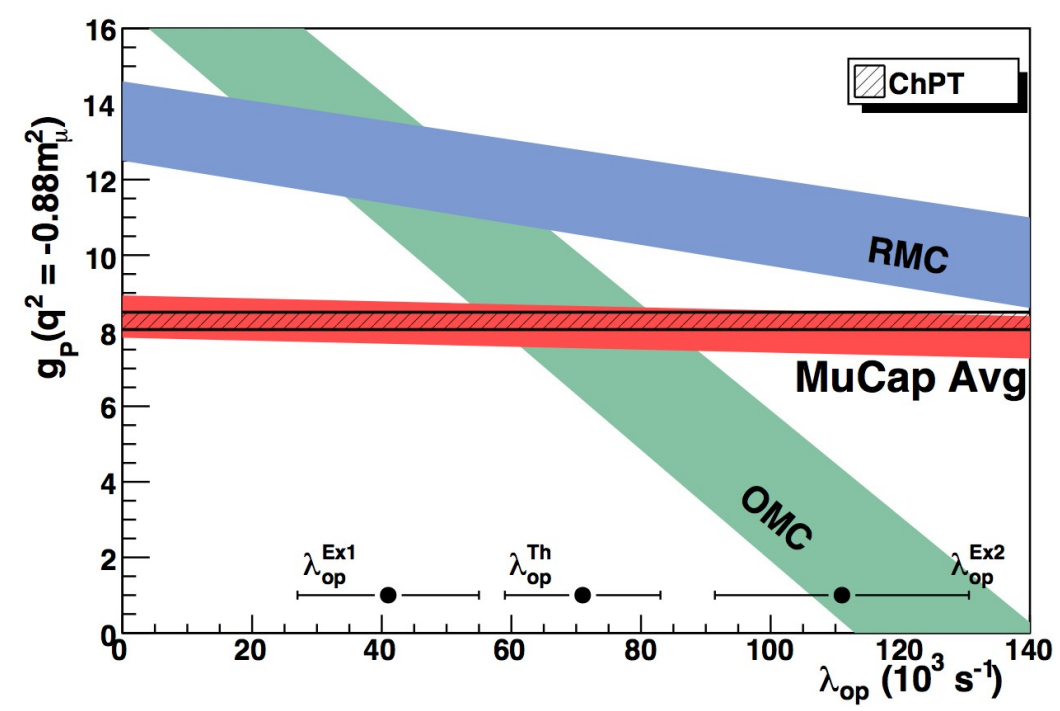

Figure 0.2: Extracted values for $g_{P}$ as a function of the poorly known molecular transition rate $\lambda_{\mathrm{op}} . \mathrm{OMC}=$ Saclay experiment [5], RMC $=$ TRIUMF experiment [17]. Also shown are results of two inconsistent $\lambda_{\mathrm{op}}$ measurements ( $\lambda_{\mathrm{op}}^{\mathrm{Ex} 1}$ from Saclay [18], $\lambda_{\mathrm{op}}^{\mathrm{Ex} 2}$ from TRIUMF [19]), and the theoretical calculation $\lambda_{\mathrm{op}}^{\mathrm{Th}}[6]$.

muons into the TPC without pile-up from second particles. This method increased the data collection rate by a factor 2 to 3 .

\subsection{Results}

During three independent production runs $[14,15] 1.2 \times 10^{10}$ fully reconstructed $\mu^{-}$decays plus $0.6 \times 10^{10} \mu^{+}$decays for systematic controls were collected. The systematic corrections include distortion effects due to impurities, removal of $\mu p$ scatter events, $\mu p$ and $\mu d$ diffusion, uncertainties of fiducial volume cuts, inefficiencies and electron track definitions. Averaging these data and using the $\mu^{+}$decay constant measured by the MuLan experiment [13], $\lambda_{\mu^{+}}=\left(455^{\prime} 170.05 \pm 0.46\right) \mathrm{s}^{-1}$, the final result of the singlet muon capture rate on the proton is obtained as [15]

$$
\Lambda_{S}^{\text {MuCap }}=\left(714.9 \pm 5.4^{\text {stat }} \pm 5.1^{\text {sys }}\right) \mathrm{s}^{-1}
$$

in excellent agreement with $\chi$ PT theory $\Lambda_{S}^{\chi \mathrm{PT}}=(715.4 \pm 6.9) \mathrm{s}^{-1}[16]$. From this result

$$
g_{P}^{\mathrm{MuCap}}\left(q_{0}^{2}\right)=8.06 \pm 0.48^{\mathrm{exp}} \pm 0.28^{\text {th }}
$$

is deducted [15]. This value is in agreement with $\chi$ PT (0.3).

Figure 0.2 shows $\bar{g}_{P}$ from recent experiments as function of the poorly known transition rate $\lambda_{\mathrm{op}}$. In contrast to previous experiments which were mostly carried out in liquid hydrogen, the MuCap experiment is virtually not sensitive to $\lambda_{\text {op }}$ and, thus, avoided this longstanding problem.

In a refined analysis [20] a new value for $\lambda_{p p \mu}$ was derived from the MuCap data and this led to an updated value of

$$
\Lambda_{S}^{\mathrm{MuCap}}=\left(715.6 \pm 5.4^{\text {stat }} \pm 5.1^{\text {sys }}\right) \mathrm{s}^{-1}
$$

and a change of -0.045 in $g_{P}^{\mathrm{MuCap}}\left(q_{0}^{2}\right)$. The change of the latter by only $8 \%$ of its uncertainty has no (visible) influence on Figure 0.2. 


\subsection{Outlook}

The determination of $\bar{g}_{p}$ from both theory and experiment requires the input of the axial vector charge radius squared $r_{a}^{2}$. In a recent review [16] discussing the values and uncertainties of $r_{a}^{2}$ obtained by different methods, the MuCap result was re-analysed. Based on the value $r_{a}^{2}=$ $(0.46 \pm 0.22) \mathrm{fm}^{2}$ evaluated from neutrino-nucleon scattering data the updated MuCap result changes to $g_{p}^{\text {MuCap }}\left(q_{0}^{2}\right)=8.23 \pm 0.83$. This is in very good agreement with the updated value $\bar{g}_{p}^{\chi \mathrm{PT}}=8.25 \pm 0.25$ which is still very close to the value of the Meissner group [3,4].

Following this path the value of $r_{a}^{2}$ is now considered to contain the largest theoretical uncertainty. Fixing $\bar{g}_{p}$ to the $\chi$ PT value, the MuCap result can be interpreted as an independent measurement of $r_{a}^{2}$ : it results in the same value $r_{a}^{2}(\mu \mathrm{H})=(0.46 \pm 0.24) \mathrm{fm}^{2}$ as from neutrino scattering.

Consequently, a new MuCap experiment with greatly increased statistics would allow a testing of the nucleon axial radius at the correspondingly increased sensitivity. Such an effort would require a newly constructed apparatus using improved detector techniques and muon beam handling.

\section{References}

[1] P. Kammel and K. Kubodera, Precision muon capture, Ann. Rev. Nucl. Part. Sci. 60, 327 (2010), doi:10.1146/annurev-nucl-100809-131946.

[2] G. Colangelo and A. Signer, A theory guide for PSI experiments, SciPost Phys. Proc. 5, 005 (2021), doi:10.21468/SciPostPhysProc.5.005.

[3] V. Bernard, N. Kaiser and U. G. Meissner, QCD accurately predicts the induced pseudoscalar coupling constant, Phys. Rev. D 50, 6899 (1994), doi:10.1103/PhysRevD.50.6899, arXiv:hep-ph/9403351.

[4] V. Bernard, L. Elouadrhiri and U.-G. Meissner, Axial structure of the nucleon: Topical Review, J. Phys. G 28, R1 (2002), doi:10.1088/0954-3899/28/1/201, arXiv:hep$\mathrm{ph} / 0107088$.

[5] G. Bardin, J. Duclos, A. Magnon, J. Martino, A. Richter, E. Zavattini, A. Bertin, M. Piccinini, A. Vitale and D. F. Measday, A novel measurement of the muon capture rate in liquid hydrogen by the lifetime technique, Nucl. Phys. A 352, 365 (1981), doi:10.1016/03759474(81)90416-4.

[6] D. D. Bakalov, M. P. Faifman, L. I. Ponomarev and S. I. Vinitsky, $\mu$ capture and ortho - para transitions in mesic molecule pp $\mu$, Nucl. Phys. A 384, 302 (1982), doi:10.1016/03759474(82)90337-2.

[7] J. Egger, M. Hildebrandt and C. Petitjean, The 10 bar hydrogen time projection chamber of the MuCap experiment, Nucl. Instrum. Meth. A 628, 199 (2011), doi:10.1016/j.nima.2010.06.316.

[8] J. Egger et al., A high-pressure hydrogen time projection chamber for the MuCap experiment, Eur. Phys. J. A 50, 163 (2014), doi:10.1140/epja/i2014-14163-1, arXiv:1405.2853.

[9] I. Alekseev et al., Cryogenic distillation facility for isotopic purification of protium and deuterium, Rev. Sci. Instr. 86, 125102 (2015), doi:10.1063/1.4936413. 
[10] V. A. Ganzha et al., A Circulating hydrogen ultra-high purification system for the MuCap experiment, Nucl. Instrum. Meth. A 578, 485 (2007), doi:10.1016/j.nima.2007.06.010, arXiv:0705.1473.

[11] R. Eichler and C. Grab, The SINDRUM-I Experiment, SciPost Phys. Proc. 5, 007 (2021), doi:10.21468/SciPostPhysProc.5.007.

[12] M. J. Barnes and G. D. Wait, A 25-kV 75-kHz Kicker for measurement of muon lifetime, IEEE Trans. Plasma Sci. 32, 1932 (2004), doi:10.1109/TPS.2004.835455.

[13] D. M. Webber et al., Measurement of the positive muon lifetime and determination of the Fermi constant to part-per-million precision, Phys. Rev. Lett. 106, 041803 (2011), doi:10.1103/PhysRevLett.106.041803, arXiv:1010.0991.

[14] V. A. Andreev et al., Measurement of the rate of muon capture in hydrogen gas and determination of the proton's pseudoscalar coupling $g(P)$, Phys. Rev. Lett. 99, 032002 (2007), doi:10.1103/PhysRevLett.99.032002, arXiv:00704.2072.

[15] V. A. Andreev et al., Measurement of muon capture on the proton to 1\% precision and determination of the pseudoscalar coupling $g_{P}$, Phys. Rev. Lett. 110, 012504 (2013), doi:10.1103/PhysRevLett.110.012504, arXiv:1210.6545.

[16] R. J. Hill, P. Kammel, W. J. Marciano and A. Sirlin, Nucleon axial radius and muonic hydrogen - A new analysis and review, Rept. Prog. Phys. 81, 096301 (2018), doi:10.1088/1361-6633/aac190, arXiv:1708.08462.

[17] D. H. Wright et al., Measurement of the induced pseudoscalar coupling using radiative muon capture on hydrogen, Phys. Rev. C 57, 373 (1998), doi:10.1103/PhysRevC.57.373.

[18] G. Bardin, J. Duclos, A. Magnon, J. Martino, A. Richter, E. Zavattini, A. Bertin, M. Piccinini and A. Vitale, Measurement of the ortho - para transition rate in the pup molecule and deduction of the pseudoscalar coupling constant, Phys. Lett. B 104, 320 (1981), doi:10.1016/0370-2693(81)90134-9.

[19] J. H. D. Clark, D. S. Armstrong, T. P. Gorringe, M. D. Hasinoff, P. M. King, T. J. Stocki, S. Tripathi, D. H. Wright and P. A. Zolnierczuk, Ortho-para transition rate in mu-molecular hydrogen and the proton's induced pseudoscalar coupling g(p), Phys. Rev. Lett. 96, 073401 (2006), doi:10.1103/PhysRevLett.96.073401, arXiv:nucl-ex/0509025.

[20] V. A. Andreev et al., Measurement of the formation rate of muonic hydrogen molecules, Phys. Rev. C 91, 055502 (2015), doi:10.1103/PhysRevC.91.055502, arXiv:1502.00913. 